\title{
POUR UNE ANALYSE ÉNONCIATIVE ET TEXTUELLE DES POINTS DE VUE PERCEPTIFS EMPATHIQUES (HÉTÉRO-PERCEPTION ET PRISE EN CHARGE ÉNONCIATIVE)
}

\author{
Alain RABATEL \\ Université de Lyon 1, ICAR, France
}

\begin{abstract}
En): The paper examines verbs of perception (VP), such as to see or to watch, in perception reports (PR) - their maximal extent includes the sentence containing the VP and the subsequent sentences reunited by semantic connections of part-whole associations in which perception gains intentional values and tends towards action. The paper first describes the notion of point of view in natural language (1) and linguistics, as well as the pragmatic and enunciative framework of the analysis of perceptions (2). Furthermore, it highlights several fundamental semantic and syntactic characteristics of PR coreferential to secondary enunciators - that are different from the initial enunciator (hetero-perceptions) -, namely the semantic and syntactic inter-sentential associations in multiplied sentence (textual period), thus raising the question of the semantactic approach of the class-objects of the VP (3). Finally, the paper discusses the notion of commitment in the case of the initial speaker/ enunciator's perceptions and/ or the secondary speaker/ enunciator's perceptions; the latter acts as a second instance of validation - in parallel to the fundamental modal instance of validation that is the initial speaker/enunciator (4).
\end{abstract}

Keywords (En): hetero-perception; speaker/enunciator dichotomy; modal subject (initial and secondary); point of view; perception-action relationship; commitment and enunciative commitment; multiplied sentence («phrase démultipliée »)

Mots-clés (Fr) : hétéro-perception ; disjonction locuteur/énonciateur ; sujet modal (premier et second), ; point de vue ; lien perception/action ; prise en charge et prise en compte énonciatives ; phrase « démultipliée »

\section{Introduction}

Alors que l'on distingue soigneusement les instances d'énonciation, lorsque l'on procède à l'analyse des discours rapportés ou représentés (RABATEL, 2003, 2008), on ne fait pas de même - à l'exception toutefois de RABATEL (1997, 1998, 2003, 2008), MAËSO (2014), lorsqu'il s'agit d'analyser des verbes de perception (VP), (e.g. BENZAKOUR, 1990 ; GREZKA, 2009), comme si l'origine énonciative n'avait guère d'importance. $\mathrm{Si}$, en revanche, on prend au sérieux l'hypothèse énonciative, notamment pour l'étude des VP, on gagne à le faire en distinguant, comme le propose DUCROT (1984 : 205), le locuteur (L), source d'un acte de parole (ou d'énonciation) de l'énonciateur $(E)$, instance source des points de vue. Cette distinction permet de rendre compte des cas de conjonction et de disjonction entre locuteur/énonciateur premier $\left(\mathrm{L} 1 / \mathrm{E} 1^{1}\right)$ ou second (12/e2), en sorte qu'il ne faut pas confondre "l'énonciateur», qui est un parasynonyme du locuteur, avec l'énonciateur en tant qu'instance modale, c'est-à-dire la source à laquelle sont rapportés les choix de référenciation des objets du discours. L'énonciateur primaire - qui peut être en syncrétisme avec le locuteur primaire, lorsque le locuteur construit

\footnotetext{
${ }^{1}$ Le slash indique un syncrétisme entre un locuteur et un énonciateur; en cas d'absence, on est confronté à un PDV d'un énonciateur second (e2), non-locuteur, puisque ce PDV ne passe pas par des paroles (DUCROT, 1984 : 204-205).
}

Echo des études romanes XIII/1, $2017 \quad$ - 147 - ISSN: 1804-8358 (Online) 
des énoncés que E1 prend en charge - doit aussi être distingué des énonciateurs seconds (RABATEL, 2010). La prise en compte de cette distinction des instances est particulièrement cruciale dans les hétéroperceptions ${ }^{2}$.

La source énonciative, en tant que source modalisante d'un point de vue (spatiotemporel, cognitif, évaluatif, etc.), a des répercussions évidentes sur la référenciation ${ }^{3}$ et les valeurs modales des propositions qui contiennent des verbes de perception et leurs arguments, et, plus largement, sur l'ensemble des prédications, qui se présentent comme des apports du VP support, et sont susceptibles d'exprimer le monde perçu en fonction de la subjectivité, de l'intentionnalité du sujet centre de perspective (GOSSELIN, 2010 : 127 ; RABATEL, $2012 \mathrm{a}, \mathrm{b}), \mathrm{y}$ compris dans le cas où ce dernier est un énonciateur second nonlocuteur (BANFIELD, 1995). Cet énonciateur second (e2) peut être considéré comme un sujet modal interne, ou une deuxième instance de validation, parallèlement à l'instance modale de validation fondamentale qu'est le locuteur/énonciateur premier.

Dans ce qui suit, je me restreindrai à quelques verbes de perception (VP), du type voir ou regarder (et de leurs équivalents, apercevoir, remarquer, etc.), en confrontant mes analyses à celles de GREZKA (2009) relatives à ces mêmes verbes. D'autres choix auraient été possibles, concernant d'autres "sens» que la vue (DUBOIS, 2009 ; KleIBER, 2011 ; VERINE, 2014), pour m'en tenir à quelques références. D'une façon générale, j'inscrirai l'analyse des VP dans celle des comptes rendus de perception ou CRP (KLEIBER, 1998; MARSAC, 2006 ${ }^{4}$; RABATEL, 2003, 2008), en prenant en compte non seulement la phrase qui contient le VP, mais aussi les phrases subséquentes, parce que c'est souvent là que la perception se charge de valeurs intentionnelles et s'oriente vers l'action. Après avoir présenté rapidement la notion de point de vue dans le langage naturel (1) puis en linguistique, ainsi que le cadre théorique de mon analyse des perceptions (2), j'examinerai les CRP (3) avant d'aborder dans un dernier point la question de leur prise en charge, par le locuteur/énonciateur premier et/ou par le locuteur/énonciateur second (4).

\section{La notion de point de vue en langue naturelle}

La notion de point de vue est polysémique et complexe en langue naturelle : un point de vue, c'est d'abord un lieu surplombant qui offre une perspective large, une belle vue, naturelle ou ordonnée par l'Homme. D'emblée, la perception visuelle s'adjoint des données cognitives, évaluatives, émotives, visant souvent des fins esthétiques voire hédonistes.

\footnotetext{
${ }^{2}$ En réalité, la distinction est tout aussi importante avec les autoperceptions, car, même s'il y a continuité ontologique, il y a fréquemment des changements de position énonciative, comme par exemple entre je narrant et je narré. On touche ici à la problématique des autres de soi, voir infra, 2.1 et 4 .

${ }^{3}$ La notion de référenciation souligne la dimension linguistique de la construction des référents (RABATEL, 2012a et b).

${ }^{4}$ La notion de CRP est également utilisée par KLEIBER (1988) pour son analyse des relatives régies par un verbe de perception (RCP) et MARSAC (2006) pour les infinitives régies par un verbe de perception (ICP).
} 
Les dimensions cognitives, évaluatives et émotives se retrouvent dans l'usage courant du terme : « avoir un point de vue », c'est avoir une opinion. Mais l'opinion n'est pas un jugement (COMTE-SPONVILLE, $2001: 412-413$ ), c'est plutôt un savoir hérité et/ou qui procède de l'expérience (personnelle ou collective) - sans prendre la forme distanciée, rationalisée du jugement critique, qui transforme l'opinion, partielle et partiale, en jugement plus global, acceptable par tous. D'où les expressions «c'est ton point de vue », «c'est un point de vue comme un autre », qui soulignent, toutes, la subjectivité et l'incomplétude du point de vue. Cet emploi n'ignore pas la dimension perceptuelle, même si celle-ci est intellectualisée. Un point de vue est toujours fonction du lieu d'où l'on regarde, d'où l'on parle, des expériences prises en compte.

Cependant, CONCHE souligne à juste titre qu'une opinion philosophique n'a rien à voir avec une opinion reçue. C'est une opinion que l'on s'est faite en raison et en méditation, et qui est une conviction»(CONCHE, 2016: 134). Par là, on se rapproche de l'acception suivante. La signification de la notion de point de vue ne se réduit pas à l'opposition avec celle de jugement. Une simple requête sur l'Internet montrerait qu'il existe à foison des expressions du type : "d'un point de vue politique », «d'un point de vue économique», d'un point de vue macroéconomique », "d'un point de vue cartésien», etc. Toutes ces formulations pourraient être remplacées par les expressions « cadre notionnel » ou «paradigme théorique », soulignant que la perspective est intellectuelle, dépendante d'un certain nombre de principes ou de situations concrètes qui pèsent sur la définition de l'objet comme sur son analyse.

J'illustre ces trois acceptions du point de vue en langue naturelle à partir du verbe voir, qui présente l'avantage d'être très polysémique et comprend le plus grand nombre de classes-objets, notées ci-dessous après les énoncés, entre chevrons, suivant la typologie des classes-objets de GREZKA (2009)5. Tous ces exemples vérifient, à des degrés variables, que la perception

- repose sur une vue (une activité perceptuelle à base physiologique) ;

- va souvent de pair avec une vision (on ne parle pas de l'objet sans une intention rétrospective ou projective) ;

- présuppose que l'objet perçu est reconnu comme appartenant à une catégorie connue et partagée ;

- présuppose ou à tout le moins sous-entend que cette activité est effectuée depuis un certain point (au sens concret ou abstrait) ;

- souligne en d'autres termes que la perception est située, en donnant à la notion de situation toute sa complexité, concernant non seulement l'emplacement du sujet percevant, son ancrage temporel mais encore ses savoirs, ses croyances, ses intentions (qui pèsent sur la perception de l'objet), ses modes d'accès au savoir (direct, perceptuel ou indirect ${ }^{6}$ ) autant que les capacités physiologiques des sujets ou les contraintes que les lieux peuvent exercer sur ce qui est visible ;

\footnotetext{
${ }^{5}$ Pour une synthèse récente du lexique-grammaire, voir Gross (2015).

${ }^{6}$ Voir les travaux sur l'évidentialité ou le médiatif chez Dendale et Tasmosvski (1994) ou GUENTCHEVA (1996).
} 
- pose donc, aussi, que la perception peut être associée à des opinions, des jugements explicites ou implicites, voire déboucher sur des décisions d'agir (ou de ne pas agir).

(1) Vois-tu le clocher de la cathédrale d'Opole ? = <acuité visuelle>

(2) Apprécier le point de vue du haut de la tour de la cathédrale présuppose que tu puisses voir $=<$ capacité visuelle $>$

(3) Il a vu passer le président de l'Université d'Opole $=<$ perception visuelle passive $>$

(4) Il veut voir le président $=<$ perception visuelle active, intentionnelle $>$

(5) Du haut du clocher de la cathédrale, on voit toute la ville d'Opole et l'Oder $=<$ propriété visuelle d'un lieu>

(6) Il va voir si la porte est ouverte $=<$ vérification $>$

(7) Comte Sponville ne voit pas que l'homme, malgré ses déterminations, puisse avoir un libre arbitre $=<$ (in) compréhension $>$

(8) Je vois bien que, du point de vue de la philosophie morale athéiste de Marcel Conche, même si l'homme est déterminé par des causes, dès qu'il se détermine à partir de telle ou telle raison, il fait œuvre de libre arbitre $=<$ constat, compréhension $>$

(9) à voir la longueur de l'exemplier, on voit que le conférencier aura de la peine à s'en tenir aux limites temporelles imparties $=<$ prédiction $>$

(10) Je verrai avec Aude Grezka si nous pouvons tomber d'accord sur un certain nombre de points $=<$ considération future $>$

Les exemples (7) à (10) illustrent de loin les significations principales de la lexie point de vue. Je privilégierai donc ci-après les VP qui expriment un point de vue, car j'accorde une grande importance à l'activité perceptuelle et à ses résultats, comme dans les exemples (1) à (6), mais aussi aux entours de cette activité perceptuelle, qu'il s'agisse des intentions, des dimensions épistémiques et praxiques auxquelles l'activité perceptuelle me semble souvent liée, à observer les VP dans leur contexte, comme ce sera le cas dans les exemples (12) à (26) - entours qui n'apparaissent pas dans les énoncés fabriqués comme dans les énoncés attestés limités à la proposition contenant le VP.

\section{Le point de vue en linguistique}

Je définis comme point de vue (PDV), en linguistique, tout énoncé qui prédique des informations sur n'importe quel objet du discours, en donnant non seulement des renseignements sur l'objet (relatifs à sa dénotation), mais aussi sur la façon dont l'énonciateur envisage l'objet, exprimant ainsi un PDV sans passer nécessairement par l'expression d'un jugement explicite, comme on l'a vu en 1. Le sujet du PDV peut être un individu, un collectif, un anonyme, et il peut exprimer des PDV singuliers ou collectifs, originaux ou stéréotypés. 
Le PDV peut renvoyer, selon une conception restreinte, à l'étude des perceptions (comme je l'ai fait dans RABATEL, 1997 et 1998) ou concerner n'importe quel énoncé, dès lors que l'analyse prend en compte les choix de référenciation des objets du discours ${ }^{7}$. Si cette extension est possible, c'est parce que le PDV relève d'une problématique sémantique générale, réfutant l'hypothèse platement référentialiste et objectiviste, selon laquelle les mots seraient «des étiquettes posées sur les choses » (DUBOIS, 2009 : 16).

Cela dit, si le PDV ne requiert pas nécessairement la présence de VP, il est intéressant de penser le PDV à partir de la perception, dans la mesure où elle présente un intérêt anthropologique fondamental bien mis en valeur par LIVET (2000). En effet, la perception s'avère vitale pour discriminer entre les choses bonnes ou mauvaises pour la survie des organismes et de l'espèce, ayant été à la base de routines comportementales qui dénotent d'emblée l'association entre perception visuelle, réflexion sur «les leçons de l'expérience », élaborations de comportements réactifs et de stratégies réflexives secondarisées, plus sophistiquées, en lien avec l'action (JOUVENT, 2013 : 11-14). Les reconstructions hypothétiques des philosophes, psychologues, neurobiologistes ne doivent pas être ignorées par les linguistes, à la condition de ne pas faire du langage la traduction d'une vérité préexistante qui réduirait à néant la nature symbolique intrinsèque de la structure des langues.

Mon approche linguistique de la notion de PDV est en phase avec les convictions fondamentales de Saussure exprimées en plusieurs endroits des Écrits de linguistique générale, notamment dans «De l'essence double du langage », quand bien même j'ai soutenu ma thèse avant la découverte de ces documents, en 1996. Selon SAUSSURE (2002), la notion de PDV est constitutive d'une part de la pratique du sujet dans la langue, d'autre part devrait être aussi constitutive de toute approche linguistique, contrairement à ce qu'une lecture objectiviste naïve du langage a pu laisser penser ${ }^{8}$ :

(11) On n'est pas dans le vrai en disant : un fait de langage veut être considéré à plusieurs points de vue ; ni même en disant : ce fait de langage sera réellement deux choses différentes selon le point de vue. Car on commence par supposer que le fait de langage est donné hors du point de vue.

Il faut dire : primordialement, il existe des points de vue ; sinon, il est simplement impossible de saisir un fait de langage.

L'identité que nous avons commencé par établir, tantôt au nom de telle considération tantôt au nom de telle autre, entre deux termes eux-mêmes de nature variable, est absolument le seul fait premier, le seul fait simple d'où part l'investigation linguistique. (SAUSSURE, $2002: 19)^{9}$

L'ensemble de la matérialité langagière peut être analysé à cette aune très générale. Le PDV correspond à un contenu propositionnel $(\mathrm{CP})$, dans des prédications dont les choix de mise en mots sont hautement significatifs. Les CP ne

\footnotetext{
${ }^{7}$ Je raconte cette extension de l'analyse des PDV dans Homo narrans, car la notion de PDV, loin de se limiter aux perceptions, se rencontre dans tous les contextes sémantiques et dans tous les types de texte et genres de discours, selon des formes variables (RABATEL, 2008 : 79, 112-115).

${ }^{8}$ Voir RASTIER (2015) et UTAKER (2016).

${ }^{9}$ Voir également Cahiers Ferdinand de Saussure 12, 1954 : 57-58, (apud Benveniste, PLG $1: 39$ 40), ainsi que SAUSSURE (2002: 201) et RABATEL (2010:361-363).
} 
font pas que référer au monde de façon vériconditionnelle, ils indiquent aussi la position (axiologique, idéologique, rationnelle, émotionnelle, etc.) de l'énonciateur sur les objets du discours. Partant, les valeurs temporelles, aspectuelles, reposent sur des points de vue, notamment à travers le choix d'un point de référence superposé ou non au point d'énonciation. De même, choisir de présenter tel référent avec un déterminant indéfini ou défini, ou un démonstratif, rend compte des visées de l'énonciateur. Les choix de catégorisation (noms et verbes), de qualification (adjectifs et adverbes), de modalité et de modalisation, d'ordre des mots et des prédications, les choix de mise en relief indiquent la position de l'énonciateur par rapport aux objets du discours. De plus, l'énonciateur énonçant une prédication choisit, au plan morphosyntaxique entre modalités d'énonciation (assertion, injonction ou interrogation) et modalités d'énoncé (exclamation, négation, emphase). Il peut encore préciser la valeur modale par le choix de termes, syntagmes ou périphrases relatifs au caractère (plus ou moins) nécessaire, obligatoire, possible ou probable du procès représenté, à l'aide des auxiliaires modaux ou de leurs équivalents adverbiaux ou circonstanciels ou périphrastiques. Il peut également évaluer le procès, expliciter son évaluation par un certain nombre de commentaires métalinguistiques portant sur le contenu propositionnel ou sa modalité, qui peut être atténuée, renforcée. D'où le fait qu'on puisse rapprocher l'énonciateur de la notion de sujet modal chez Bally (DUCROT, 1989 : 181-191), dans la mesure où son empreinte se voit non seulement dans le modus, mais encore dans les choix qui organisent le dictum. Le PDV touche enfin à l'organisation des discours, y compris sous leur dimension rhétorique. Si le PDV se repère le plus souvent dans une prédication, il est néanmoins possible qu'il se repère dans le choix d'un seul terme dont la dénomination est sentie comme problématique, et il peut aller jusqu'à compacter en une sorte de méta-PDV un grand nombre de prédications qui traitent du même thème et/ou de thèmes approchants selon une même vision ou orientation argumentative (RABATEL, $2008: 67-73$ ).

\subsection{Les différentes formes de PDV empathique}

Mes travaux sur le PDV concernent surtout la possibilité que se donne un locuteur de changer de position énonciative en se mettant à la place d'un autre : cet autre, présent dans une prédication ${ }^{10}$, est le plus souvent un autre que soi (soi étant égal à L1/E1), en contexte hétérodialogal et/ou hétérodialogique, mais c'est aussi, en contexte autodialogique, un autre de soi; cela dit, comme je vais traiter d'hétéroperceptions, ce sont les autres que soi qui m'intéressent ici. Dans tous les cas, cette conception de l'altérité conjoint PDV et empathie (RABATEL, 2008, $2014)^{11}$. Avant d'illustrer le phénomène, je rappelle brièvement que la notion

\footnotetext{
10 On peut encore définir le point de vue comme « un contenu propositionnel renvoyant à un énonciateur auquel le locuteur "s'assimile" ou au contraire dont il se distancie » (RABATEL, 2005).

${ }^{11}$ Un des points communs entre empathie et PDV repose sur l'aptitude au décentrement et ce que j'appelle la mobilité empathique, c'est-à-dire l'aptitude à changer non seulement de sources du PDV en se mettant à la place d'un autre, mais aussi celle qui consiste, tout en restant soi-même, à être capable de changer de perspective, par exemple en réfléchissant à une situation en l'analysant d'un point de vue économique, financier, politique, social, culturel ; en réfléchissant à un concept d'un point de vue théorique (et en convoquant diverses approches théoriques), d'un point de vue pratique (et en
} 
d'empathie - ou empathie secondaire, par opposition aux étapes pré-empathiques (BRUNEL et COSNIER, 2012) - se définit par l'aptitude à se mettre à la place des autres. L'empathie a une dimension imaginative, projective, de nature essentiellement cognitive, sans exclure une dimension affective, émotionnelle (voir BERTHOZ, 2004 ; TISSERON, 2010). Si l'on déploie ce que signifie « se mettre à la place des autres $\gg-$ en prenant la notion de place soit au sens spatial, soit en un sens plus abstrait, notionnel, on dira qu'on peut imaginer ce que les autres perçoivent (avec leurs sens), ressentent (selon leurs affects, émotions, sentiments), pensent, disent (avec toutes les formes de discours représentés), font. En réalité, la formulation précédente est trop platement descriptive, il faudrait y intégrer une dimension modale qui consiste à s'interroger sur ce qu'un autre peut, veut, doit, sait (et de même à la forme négative) percevoir, ressentir, penser, dire ou faire. Ces phénomènes psychologiques peuvent être étudiés en linguistique, ce que je me suis particulièrement attaché à faire en analysant l'expression linguistique des perceptions, des affects, émotions et sentiments, des pensées et des paroles, des actions, selon des continuums allant du sensible à l'intelligible et au praxique, et, surtout, en distinguant ces divers PDV selon leur source énonciative, l'énonciateur primaire ou des énonciateurs seconds, comme le montre l'analyse de l'exemple (12), que je choisis parce qu'il aligne successivement les trois principales " modalités » ou types de PDV que j'ai distingués, les PDV embryonnaire, représenté et asserté.

(12) 17, 42 Le philistin regarda et, quand il aperçut David, il le méprisa : c'était un gamin au teint clair et à la jolie figure. 43 «Suis-je un chien pour que tu viennes à moi armé de bâtons ? » (1 Samuel, 17, 42-43, TOB : 542)

En (12), ces 3 formes de PDV coréfèrent au sujet percevant second qu'est le philistin, c'est-à-dire Goliath, et non à la perception du locuteur/énonciateur premier (L1/E1), le narrateur, qu'on peut, pour simplifier, considérer comme la trace intratextuelle de l'auteur, le prophète Samuel. Le PDV embryonnaire (souligné) est au PS, et comprend trois verbes, dont deux de perception, « regarda » et « aperçut ». Le lien entre la perception intentionnelle et le procès mental de mépris est quasi immédiat, dans la mesure où la valeur du « quand »-qui pourrait être remplacé par un « (aus)sitôt que, dès que »-indique une quasi concomitance entre la perception et la réaction de mépris (ce que confirme la version originale). L'exemple (12) montre d'une part que, contrairement à ce que dit Benveniste, la narration au PS n'est pas toujours objective, et qu'elle est compatible avec la subjectivité d'un énonciateur second sujet d'un énoncé en il (OLSEN, 2002 ; RABATEL, 2008). Le procès de perception au passé simple renvoie à la perception globale de l'énonciateur second (mais déjà subjective). L'exemple confirme d'autre part que la perception physio-cognitive peut cumuler en concomitance perception visuelle et cognition : en effet, « aperçut » pourrait être remplacé par voir, le voir 9 de GREZKA de $<$ perception physio-cognitive $>^{12}$.

se mettant à la place d'usagers très différents), éventuellement en prenant en compte ses propres évolutions théoriques ou pratiques, etc. Voir RABATEL $(2008,2014 \mathrm{~b})$ et $(2016: 143-145)$.

${ }^{12}$ Exemple : « Je vois que tu as cassé le vase ». Selon GREZKA, ce voir 9 est proche du CR de perception visuelle passive, mais diffère par la non-simultanéité temporelle, puisqu'il y a un écart entre la 
Le PDV représenté, en italique, émerge par opposition au PDV embryonnaire, avec lequel il est en fort contraste, du fait de l'opposition entre le premier plan, avec le temps prototypique du passé simple, et l'imparfait, qui présente le procès comme s'il était expériencié, de l'intérieur de la subjectivité du sujet percevant : s'installe donc ici un discordanciel énonciatif ${ }^{13}$, propice à l'expression d'un phénomène perçu d'abord globalement et de l'extérieur, pour ainsi dire (« il le méprisa »), tandis que le fragment en italique expanse l'impression première de mépris en détaillant les aspects de David susceptibles de justifier le jugement de Goliath. Outre les deux points qui ouvrent sur un commentaire explicatif, le présentatif (RABATEL, 2001b), avec sa visée sécante, présente une perception intériorisée, qui cumule des éléments perceptifs apparemment objectifs (et qui sont d'ailleurs objectifs en ce sens que si nous avions à nous représenter David, ou à mettre en scène l'épisode, de fait David devrait être jeune, avoir l'air juvénile, un teint clair, être gracieux voire gracile) et des jugements accolés à ces descriptions, et ce, malgré l'absence de jugement explicite. Premier jugement : "c'était un gamin »: le terme est péjoratif et les inférences permettent de comprendre que Goliath s'offusque de voir en face de lui, en tant que champion des juifs, un jeune adolescent, et non pas un homme mûr, à son image. Le contraste est encore plus fort du fait de la grande taille de Goliath. Circonstance aggravante, c'est un gamin « au teint clair et à la jolie figure » : ces qualificatifs positifs sont en principe réservés au physique féminin, aussi leur utilisation pour un homme leur donne-t-elle un tour méprisant.

Enfin, le PDV asserté est exprimé dans le fragment graissé, qui correspond à du discours direct : c'est donc le seul PDV dans lequel l'énonciateur second est aussi locuteur second. Le PDV verbalise dans une montée de la colère toute l'indignation de Goliath, offusqué de n'avoir pas un adversaire digne de son rang. Ainsi, la description est fortement subjectivisée, et le narrateur tente de reproduire empathiquement le PDV très stéréotypé et machiste de Goliath. Cette reconstruction empathique n'est pas synonyme de sympathie, puisqu'à l'évidence, ceux qui connaissent l'épisode de près savent bien que le récit (ou le narrateur) empathise globalement sur David et est en consonance sympathique avec lui : mais même si le lecteur ne connaît pas le texte, ni son contexte, il lui suffit de repérer l'opposition des appellatifs pour comprendre la proximité du narrateur avec David, nommé par son prénom, tandis que son ennemi est nommé par son ethnonyme, marqueur de distance.

Mais ici, il y a un relai d'empathisation provisoire sur la façon de penser de l'adversaire. Dernière remarque, capitale au plan méthodologique et épistémologique : le PDV représenté ne comprend pas de terme de perception. Il

perception et la prédication externe, souvent dans une complétive, qui se présente comme le résultat de la perception (GREZKA, $2009: 178-179)$ : e.g. Je n'a pas forcément vu le moment où le vase a été cassé (voire qui l'a cassé : «j'ai vu que le vase est cassé »). Il s'ensuit qu'il n'y a pas de subordonnée infinitive ou relative avec V9, puisque l'infinitive et la relative supposent la simultanéité (GREZKA, 2009 : 180) : certes, l'analyse est correcte pour l'exemple cité, mais elle ne cadre pas avec l'exemple (12) ci-dessus.

${ }^{13}$ Ici, le discordanciel énonciatif n'implique pas un changement de locuteur, mais un changement dans la façon dont ce dernier, en tant qu'énonciateur, change de position énonciative, en voyant les choses synthétiquement puis analytiquement. 
évoque pourtant le résultat d'une activité de perception, ce qui implique que la perception doit s'appuyer d'une part sur ce qui est explicite et d'autre part sur les inférences, sur l'implicite. Toute analyse globale des perceptions gagne à cumuler les perspectives sémasiologiques et onomasiologiques. Les deux perspectives sont légitimes, et, surtout, gagnent à être articulées, sauf à laisser de côté des pans entiers de la perception, tant sous son versant ontologique, phénoménologique, que sous son versant linguistique, qui ne peut être limité à la seule analyse des mots reconnus comme exprimant la perception...

\subsection{Les PDV et les comptes rendus de perception}

Si le PDV concerne tous les contenus propositionnels (CP), indépendamment de leurs contenus sémantiques, il est malgré tout intéressant de s'arrêter, parmi ces $\mathrm{CP}$, sur ceux qui sont des comptes rendus de perception (CRP), à l'instar de ceux qui figurent dans le verset 42 de l'exemple de Samuel ou dans les exemples cidessous, qui sont passibles d'une analyse sémasiologique et onomasiologique.

Comme pour l'exemple précédent, le PDV représenté est en italique, le PDV embryonnaire est souligné ${ }^{14}$. Les PDV comprennent des perceptions, auxquelles s'ajoutent des émotions, des jugements de valeur implicite, des pensées de nature inférentielle greffées sur les perceptions, sans passer par des formes traditionnelles de discours rapporté : c'est donc cet ensemble hybride qui forme des CRP. Ces derniers sont souvent annoncés par un verbe de perception dans le premier plan, éventuellement couplé à un verbe de mouvement (13) ou à un verbe statique (14), (15) qui indiquent en contexte une prédisposition à regarder. A défaut de verbe, un nom peut indiquer ou présupposer une activité de perception, comme « spectacle », en (16). De plus, le verbe de perception peut être sous-entendu par la situation, comme en (17): la fenêtre (un topos du roman réaliste) renvoie à des scénarios mondains propices à une activité de perception, en sorte que ce qui suit est rapporté à la perception de Jeanne. Parfois, le CRP ne présente que le résultat de la perception, comme en (18), sans mention préalable d'un verbe de perception, ni d'un autre verbe présupposant une activité de perception, a fortiori sans mention

${ }^{14} \mathrm{Il}$ y a PDV embryonnaire, ce qui signifie que les énoncés ne sont pas « objectifs » (contrairement à ce qu'affirmait Benveniste), mais expriment d'emblée le PDV du personnage : ainsi, en (17), par inférence à reculons, et compte tenu du mais argumentatif, la première proposition n'est pas un simple énoncé constatif, mais exprime une modalité désirante contrariée. Car si Jeanne s'approche de la fenêtre après avoir terminé ses malles, c'est pour voir si elle peut enfin partir, puisque la fin de la phrase indique ( ne cessait pas ») que Jeanne savait qu'il pleuvait avant même d'avoir fermé ses malles. On pourrait mutatis mutandis faire partout la même analyse. C'est ce qui explique que l'énoncé puisse être lu selon deux registres différents, relativement objectif du point du narrateur, censé rapporter fidèlement une réalité (vue à travers un état de conscience) et subjectif (du point de vue de cet état de conscience) : ainsi, en (17), les éléments du décor sont vrais ; et Jeanne est dépitée. En (14), l'usine correspond bien, « objectivement », à la description qui en est faite, mais aussi elle exprime le PDV «un peu déprimé » de Chauvieux, formulation litotique qui indique au contraire que le personnage l'est beaucoup, si l'on prend en compte la masse des informations qui peuvent connoter la dépression (RABATEL, 2015: 126-127). Bref, dans le PDV embryonnaire, la perception est appréhendée globalement, comme un tout ou un point (du fait du PS ou de tout autre tiroir verbal d'aspect global), tandis que la perception dans le PDV représenté détaille la perception, vue de l'intérieur du sujet de conscience/sujet modal. Selon la présence de subjectivèmes ou non dans le PDV embryonnaire, ce dernier sera objectivant ou subjectivant. 
d'un sujet percevant. Cependant on peut faire l'hypothèse que le fragment en italique, compte tenu des choix de référenciation, correspond à la perception d'un sujet percevant subissant une forte attraction/répulsion pour les intellectuels (sans en être un lui-même), comme le laissent d'emblée penser la dénomination méprisante, "l'intellectuel», avant les commentaires décalés sur «viande de femme », en appui sur une catégorisation elle-même inattendue du corps/teint ou de la peau comme « chair ».

(13) Elle sauta du lit et il la regarda traverser la chambre d'un pas résolu. Elle est trapue, les fesses sont carrées, les hanches droites, la poitrine musclée; ces corps-là ne s'abîment pas, le visage se fane avant que les seins ne tombent. (Vailland, Les mauvais coups, Livre de poche: 9)

(14) Chauvieux arriva à l'usine un peu déprimé. Il s'arrêta une minute à l'entrée pour considérer les trois groupes de bâtiments en $\mathrm{W}$, entre lesquels étaient ménagées deux allées étroites, bordées de fleurs chétives. La lumière de l'été mettait en valeur les arêtes vives et austères de ces vastes hangars vitreux. Il ne percevait rien de l'activité des ateliers qu'un bruit de machine, étouffé et profond et qui semblait la respiration d'une ville endormie. (Aymé, Travelingue, Folio : 106s)

(15) $\mathrm{Il}[=\mathrm{Glen}] \mathbf{s}$ 'arrêta au milieu du cimetière et jeta un regard circulaire. Puppy avait dit pas loin de tante Eva, mais il n'était pas sûr de savoir où elle était. Ca faisait si longtemps qu'elle était morte. L'enterrement d'Eva était une vieille histoire, il s'en souvenait à peine. Des gamins avec des cravates, des femmes en pleurs, et de la boue sur les chaussures. Il était petit à l'époque. Un Davis, ou un Clark, elle serait près d'eux. Il se mit à lire les noms en progressant vers la droite, puis il se trouva brusquement parmi eux. Ils étaient tous enterrés ensemble, et cela depuis cent ans. Les pères, les mères, les enfants, les grands-pères et les victimes de trois guerres. Il trouva la tombe, et il fut stupéfait. Pas de pierre tombale, rien qu'un petit écusson en métal avec une carte blanche fixée dessus par une pince. Le nom de la société de pompes funèbres était marqué en relief, et c'était ce qui désignait l'endroit où elle reposait. (L. Brown, Père et fils, Gallimard La Noire 1999 : 17)

(16) Le commissaire s'éloigna vers le boulevard, où était garée sa voiture. Rovère descendit la rue Sainte-Marthe, entraînant Dimeglio dans son sillage. Le spectacle $n$ 'était guère réjouissant. Des gosses au nez souillé de morve jouaient sur les trottoirs, juchés sur des skates bricolés; ils zigzaguaient avec une étonnante maestria entre les poubelles et les tas de gravats. Une file de crève-la-faim attendait devant une boutique, tenue par une certaine Mission Evangélique, où l'on servait un bol de soupe. De nombreuses fenêtres murées par des parpaings aveuglaient les façades où de larges trouées de couloirs sombres, pareilles à des soupiraux, jaillissaient à ras de la chaussée, et laissaient entrevoir des conduites de gaz éventrées, des amas de fils électriques déchiquetés, et plus loin, dans les courettes dont ils révélaient l'existence presque à contrecœur, un fouillis d'ordures, une quincaillerie sauvage amassée là sans motif avouable. (Jonquet, Les orpailleurs, Série Noire Gallimard : 28)

(17) Jeanne, ayant terminé ses malles, s'approcha de la fenêtre, mais la pluie ne cessait pas. (Maupassant, Une vie, Folio : 27)

(18) L'intellectuel a enlevé sa chemise : sa chair est blanche; c'est de la viande de femme. (Bory, Mon village à l'heure allemande, J'ai lu : 115)

Les CRP présentent donc des perceptions appréhendées globalement tandis que d'autres, dans des énoncés, le plus souvent postérieurs à la perception globale, détaillent cette dernière, dans des anaphores associatives avec des facettes de l'objet 
perçu et des traces cognitives, émotives ou axiologiques du sujet relatives à sa perception de l'objet (RABATEL, $1998: 54$ ).

Avant de faire un premier bilan sur ces PDV associant perceptions, pensées (et/ou paroles) et action, je voudrais répondre d'emblée à l'objection que ma théorisation serait influencée par mon corpus de textes narratifs littéraires. Cette influence est indéniable: les récits, orientés vers l'action, expliquent que les perceptions soient associées à des pensées (sous la forme de pensées explicites ou d'inférences), le tout motivant les (ré)actions. Néanmoins, cette situation a une dimension anthropologique générale (cf. supra les références à Livet ou Jouvent) qui n'est pas propre aux textes littéraires narratifs, tant s'en faut. Partant de là, toute la question, fortement posée par le cadre théorique pragma-énonciatif du PDV, est de savoir s'il faut borner l'analyse des perceptions à la proposition ou à la phrase qui comprend le(s) terme(s) de perception, ou s'il faut au contraire prendre en compte les phrases qui précèdent ou qui suivent, et jusqu'où. Cette question interroge les limites de la syntaxe et la nature des liens textuels de nature transphrastiques qui organisent les CRP. Bien évidemment, cette interrogation n'est pas sans lien avec une des conclusions partielles précédentes, relative à la nécessité de cumuler approches onomasiologique et sémasiologique. Je voudrais, dans les parties suivantes, avancer quelques arguments en faveur de cette thèse.

\section{Les verbes de perception dans les comptes rendus de perception (CRP)}

Dans cette section, je ne me limiterai pas à la phrase ou à la prédication/proposition contenant le VP, je traiterai globalement de la perception à l'échelle du texte, en tenant compte de la dynamique interprétative construite par le caractère global, diffus, de comptes rendus de perception qui déborde du cadre propositionnel, voire de la phrase complexe ou multiple.

\subsection{De la perception passive, non intentionnelle à la perception active, intentionnelle : la perception et ses enjeux actionnels}

Le premier enseignement est que la distinction VP active (prototype : regarder) ou passive (prototype : voir) est bien sûr importante, mais qu'en discours, elle est à relativiser, dans de nombreux cas. En effet, lorsque le CRP part d'une perception passive (ou non intentionnelle), prédiquée une fois, globalement puis s'ouvre sur des propositions qui détaillent, approfondissent les caractéristiques de l'objet perçu, il se charge au fil du texte d'une dimension interprétative, attribuée à la source percevante, passant ainsi d'une perception passive, involontaire, à une perception active et intentionnelle. Mais c'est une intentionnalité d'aval, résultant d'inférences à reculons, sur la base des instructions du texte. Par conséquent, ce qui est d'abord vu passivement, entrant dans le champ visuel du sujet percevant de façon non intentionnelle, peut ensuite devenir une perception active, intentionnelle en ce que le sujet percevant cherche à comprendre la logique de ce qu'il perçoit, comme on le voit dans l'exemple suivant, où Fabrice, après avoir perçu un bruit, met en relation sa source avec les effets mortels qu'elle produit.

(19) Il entendit un bruit sec auprès de lui : c'étaient deux hussards qui tombaient atteints par des boulets (Stendhal, La Chartreuse de Parme, apud Rabatel 1998 : 72) 
Le mouvement va du non intentionnel et du passif vers l'intentionnel et l'actif - l'inverse est difficile (GREZKA, 2009 : 12), lorsque le VP est associé en amont ou en aval à des verbes de mouvement ou des situations qui présupposent mouvement et attention (voir infra (20), (21), (22), (23)), ou encore lorsque le co(n)texte comprend des traits perceptifs et cognitifs qui dénotent non seulement une acuité perceptive mais une capacité d'analyse des percepts (voir (19), (22)). Le passage d'une perception passive à une perception active est favorisé par la situation statique qui facilite une perception analytique après une première phase de perception synthétique globale et passive, sans compter la prise en compte de la situation des sujets percevants, de leur psychologie, de leur sociologie, de leur histoire, de leur idéologie, ou encore celle des enjeux événementiels, actionnels où ils se trouvent pris (voir supra (13)-(15) et infra (24), (26)).

C'est pourquoi, en (19), on a un VP passif et non intentionnel immédiatement suivi, après les deux points et dans un énoncé avec un imparfait à valeur commentative et explicative, du résultat analytique du procès de perception antérieur. Cette activité perceptive active et intentionnelle est d'autant plus motivée que le contexte de la bataille oriente la perception vers l'action, pour choisir l'emplacement le plus favorable pour protéger sa peau. Il en va de même, mutatis mutandis, dans l'exemple (20), même si le verbe de perception se voir indique bien d'abord une activité physiologique, qui peut être passive ou active, intentionnelle ou non - sans compter une dimension représentationnelle qui n'est pas activée ici.

(20) Albert entrebâille la fenêtre. Edouard le suit des yeux. Le jeune blessé insiste, "plus grand », il fait signe des doigts, «non, moins », « un peu plus », Albert s'exécute, écarte davantage le vantail, et, quand il comprend, c'est trop tard. a force de chercher sa langue, de s'écouter proférer des borborygmes, Edouard a voulu savoir : il se voit maintenant dans la vitre.

L'éclat d'obus lui a emporté toute la mâchoire inférieure; en dessous du nez, tout est vide, on voit la gorge, la voûte, le palais, et seulement les dents du haut, et en dessous, un magma de chairs écarlates avec au fond quelque chose, ça doit être la glotte, plus de langue, la trachée fait un trou rouge humide...

Edouard Péricourt a vingt-quatre ans.

Il s'évanouit. (Lemaître, Au revoir là-haut, Albin Michel 2013 : 78-79)

En contexte, la longue description en italique de la découverte qu'il est une gueule cassée traduit un auto-examen attentif, par le héros, ce que confirme le «maintenant» qui se rapporte à l'actualité du sujet percevant. Cet examen en focalisation interne suit sa découverte horrifiante des dégâts, privilégiant l'hypothèse active, tout comme les requêtes antérieures le donnent à entendre («le jeune blessé insiste, «plus grand », il fait signe des doigts, «non, moins », « un peu plus » ») et confirme constamment l'hypothèse intentionnelle, marquée par « Edouard a voulu savoir ». Au fond, l'entour du verbe, avec la modalisation du vouloir savoir et l'adverbe indique, dans le co-texte étroit du VP, ce que le co-texte élargi confirme, en rendant d'emblée indissociables la perception physiologique de ses motivations et de ses composantes cognitives et, en l'occurrence, émotives. Mais à tout cela s'ajoute encore une dimension actionnelle, même si elle s'exprime paradoxalement par un "évanouissement»: car le héros mesure désormais que 
toute action ordinaire de la vie ordinaire d'un homme ordinaire est désormais interdite à l'atroce gueule cassée qu'il est devenu.

\subsection{Prédications secondes parataxiques implicitement subordonnées à une prédication première dans une phrase précédente: vers la phrase «démultipliée » ou la «période-textuelle» pragma-énonciativement homogène}

D'un point de vue formel, l'exemple (20) est complexe, relativement à l'analyse des liaisons de prédications, parce qu'on ne peut s'arrêter à l'analyse de la phrase : ici, il y a bien un COD («il se voit »). Mais ce qui suit la phrase contenant le verbe de perception peut aussi être considéré comme sous la rection du verbe voir (et non plus se voir ${ }^{15}$ ), comme si l'on avait quelque chose comme: "Il voit que l'éclat d'obus lui a emporté toute la mâchoire inférieure, $q u$ 'en dessous du nez tout est vide, $q u$ 'on voit la gorge », quand bien même voir n'y a pas partout la même signification ni n'appartient à la même classe-objet, notamment dans « il voit (comprend) qu'on voit (perçoit visuellement) la gorge $» . .$.

Par conséquent, toutes les propositions de la phrase qui commence avec «L'éclat d'obus » jusqu'à «la trachée fait un trou rouge humide» peuvent être considérées comme étant à la croisée de phénomènes complexes analogues : d'une part à la « juxtaposition coordonnante » analysée par ROIG et VAN RAEMDONCK, dans le cadre d'une phrase multiple, d'autre part à l'hypotaxe paradigmatique, dans le cadre de la phrase complexe (ROIG et VAN RAEMDONCK, 2014 : 16-18). Je dis « analogue », car on est ici au-delà de la phrase multiple et de la phrase complexe. La description qui cherche à mettre des noms sur le résultat de cette perception/auscultation ( $"$ avec au fond quelque chose, ça doit être la glotte ») relève de prédications secondes de même niveau parataxique entre elles, et c'est en ce sens que ces phrases relèvent d'une sorte de juxtaposition coordonnante, bien qu'elles débordent du cadre de la phrase multiple. De plus, comme ces propositions sont sémantiquement «subordonnées » (si l'on peut dire) à la phrase précédente avec «se voit», il est légitime d'évoquer un phénomène analogue à l'hypotaxe paradigmatique, quoiqu'on ne soit pas dans le cadre de la phrase complexe, que ROIG et VAN RAEMDONCK nomment «juxtaposition enchâssante » ou « enchâssement implicite » lorsque le lien de hiérarchisation ne passe pas par un outil d'enchâssement. Cette "subordination syntaxique interphrastique » passe par une mise en discours qui sous-entend un verbe de perception, présuppose un procès de perception rapporté au personnage, et repose sur une autonomie sémantique toute relative $^{16}$ : certes, les propositions de la phrase multiple sont autonomes, mais elles

\footnotetext{
${ }^{15}$ Encore que ce serait possible, à la condition que se voit soit repris par un verbe du type apercevoir, constater: «Il se voit avec l'éclat d'obus qui lui a enlevé toute la mâchoire inférieure, s'aperçoit qu'en dessous du nez tout est vide ».

${ }^{16}$ Toutes choses égales, on observe un même paradoxe chez MARSAC (2010) lorsqu'il analyse les liens linguistiques dans des phrases du type «J'entends les oiseaux chanter», relativement au statut fonctionnel de l'infinitif régi par un VP, dans la mesure où la syntaxe postule deux compléments, la sémantique un argument, et où il propose de traiter ce genre d'énoncé comme un actant complétif direct fragmenté en deux parties solidaires qui ne constituent pas deux actants distincts, plutôt que de les analyser en prédication première et seconde. Mais cette analyse fait particulièrement sens dans le cadre phrastique, la question des liens de prédications est plus complexe dans un cadre interphrastique.
} 
ne font vraiment sens que rapportées à l'intention première de l'auscultation, suivant les relations, déjà évoquées, de l'anaphore associative. La phrase multiple peut, à cet égard, être interprétée comme une prédication seconde par rapport à la phrase antérieure, comme des apports d'informations à la phrase support antérieure. Bref, on n'est pas ici dans le domaine des phrases multiples, c'est pourquoi je risque les néologismes de «phrase démultipliée », de " période textuelle d'un empan plus large que la phrase », mais qui est organisée en ayant la mémoire de l'empreinte phrastique, en sorte qu'on a une période textuelle d'après " l'invention de la phrase ", fortement homogène au plan pragma-énonciatif, alors même que «les marques " «s'estompent», comme le disent ROIG et VAN RAEMDONCK, non seulement dans le cadre intraphrastique, mais dans le cadre interphrastique.

L'exemple (21) pourrait faire l'objet d'une analyse analogue ;

(21) Il se leva $\mathrm{P}-1$, vit par la fenêtre la forêt blanche à perte de vue $\mathrm{P} 0$, et se recoucha dans la chambre quiète avec un contentement qui lui faisait cligner les yeux P1. Le silence respirait autour de lui plus subtil sous cette lumière luxueuse P2 (Gracq, Un balcon en forêt, apud Maëso 2014 : 133)

Apparemment, le VP porte sur le COD « forêt blanche », et l'on pourrait penser qu'il n'y a pas de rapport entre P0 (qui contient le VP dans la notation de Maëso, $\mathrm{P}-1$ équivalant à la phrase antécédente et $\mathrm{P} 1, \mathrm{P} 2$, aux phrases subséquentes) et $\mathrm{P} 1$, $\mathrm{P} 2$, encore que cette hypothèse tiendrait pour nulle la continuité référentielle des anaphores pronominales. Certes, il n'y a pas de rapport explicite entre la perception et le sentiment de «contentement » en P1 ; mais le clignement « des yeux » est dû autant au contentement, comme cela est dit explicitement, qu'à la blancheur « à perte de vue », en sorte qu'il y a un lien implicite entre la perception et le sentiment qu'elle provoque. De même, s'il n'y a pas de relation syntaxique explicite entre P0 et P2, la synesthésie du spectacle de la blancheur illimitée et du silence - si l'on prend en compte « la lumière luxueuse », qui renvoie à la blancheur (cf. « lumière ») et à « contentement » (cf. « luxueuse ») - alimente l'hypothèse que P2 est du même niveau syntaxique que P1, exprime comme P1 une même euphorie sous la rection implicite du VP de $\mathrm{P} 0$. Bref, là encore, le VP voir signifie bien en $\mathrm{P} 0$ une perception physiologique directe, mais il signifie en discours, dans $\mathrm{P} 1$ et $\mathrm{P} 2$, une perception associée à de la cognition et aux émotions/affects/sentiments, quand bien même le VP est seulement perceptuel, physiologique, bien différent du verbe voir qui associe perception physique et cognition comme dans « Il vit tout de suite que Pierre était mal à l'aise ». D'ailleurs, (21) pourrait être réécrit de façon à mettre en relief ces liens de juxtaposition coordonnante (entre P1 et P2) et d'enchâssement implicite (entre P0 et P1-P2) :

(21b) Il se leva P-1, vit par la fenêtre la forêt blanche à perte de vue P0. Un contentement lui faisait cligner les yeux $\mathrm{P} 1$. Le silence respirait autour de lui plus subtil sous cette lumière luxueuse P2

$\mathrm{Si}$, dans le cas des exemples qu' analyse Marsac, on comprend qu'on puisse rechercher une explication syntaxique qui fasse l'économie de l'explication par la relation prédications première, seconde, il en va différemment dans les exemples que j'analyse. 
Ce qui précède envisage l'unité pragma-énonciative interne des séquences, appréhendées du point de vue d'une même source, celle-ci pouvant varier de formes de PDV. Il va de soi que cette unité, redoublée par la cohérence sémantique du fragment, par ces phénomènes de marquage de la subjectivité de nature transphrastique, qui fonctionnent comme des marques internes des PDV, peut aussi être analysée du point de vue de ses marques externes, qui posent la question du début et de la fin de ces séquences. Je n'aborde pas cette question, dont j'ai traité dans RABATEL (2008 : 73-79), à travers la question du bornage externe du PDV, qui passe souvent par des changements de régime narratif, avec retour à la narration, à des formes de premier plan, conjointes à des changements de situation, de personnages, ou d'orientation argumentative, etc., comme c'est le cas en (20).

\subsection{Quand la saisie de la mise en discours complexifie l'analyse des classes- objets de la perception dans le cadre du lexique-grammaire}

L'approche du lexique par les classes-objets du lexique grammaire, que met en œuvre Grezka pour les VP, débouche sur des sous-classes sémantiques (ou sémantaxiques), préférées à l'approche par les relations thème-rhème, abandonnant la représentation bipolaire de la phrase élémentaire pour une conception atomique, autour du verbe, avec ses éventuels arguments, spécifiés selon leur rôle syntaxique (GREZKA, 2009 : 17-23). Ce choix, tout à fait compréhensible, a un double coût, relativement à la question de la référence et à celle de la mise en discours. Car l'actualisation des arguments est indifférente à la mise en discours : de fait, $L u c$ aime Léa et Luc éprouve de l'amour pour Léa correspondent au même schéma d'argument pour un prédicat de premier ordre (essentiel à la phrase, cf. compléments essentiels) qui peut prendre une forme verbale, nominale, voire adjectivale (Luc est amoureux de Léa) : bref ces 3 formes ne modifient pas la structure sémantique du contenu prédicatif ${ }^{17}$. Or, j'ai tenté de montrer plus haut que la mise en discours, au plan interphrastique, pesait sur l'interprétation et sur les analyses syntactico-sémantiques, par delà la structure argumentale des VP, compte tenu des relations discursives interpropositionnelles et interphrastiques.

De nombreux facteurs de la mise en discours complexifient l'analyse des VP. Ces facteurs peuvent être internes à la phrase contenant les Vp, comme les adverbes en (22), le tiroir verbal en (25), la modalisation du verbe en (26); mais ils peuvent aussi être externes à la phrase et figurer dans le reste du CRP, comme dans les exemples (23), (24) et (26). En fait, si la distinction est utile, il est plus important encore de mesurer que les facteurs qui pèsent sur le sens du VP fonctionnent souvent de conserve, et dans la phrase contenant le VP et dans les phrases suivantes (c'est pourquoi je renonce à classer séparément les exemples ci-après).

Ainsi, le sens et la valeur du verbe sont modifiés en (22) en raison des deux adverbes qui accompagnent le verbe entendre:

\footnotetext{
${ }^{17} \mathrm{NB}$ : pour les substantifs, il faut distinguer ceux qui s'analysent comme prédicats ou bien comme arguments élémentaires (qui ne peuvent jamais être prédicatifs) : construction est prédicatif dans L'entreprise a procédé à la construction du bâtiment (= a construit le bâtiment). Dans La construction est haute de 50 mètres, construction est en position argumentale. Ces arguments peuvent devenir prédicats dans d'autres constructions (GREZKA, 2009 : 23-24).
} 
(22) La meute contourna silencieusement un massif rocheux couvert de ronces et se dirigea vers un chêne à l'énorme tronc noueux. Orientant sans cesse ses oreilles, différenciant les bruits, situant leur origine et leur éloignement, Bien-Noir entendit presque simultanément un campagnol s'enfuir à toutes pattes sur le sol feuillu, les griffes d'un écureuil remontant précipitamment en haut de son arbre, le chuintement d'une limace rampant non loin sur un champignon, les grattements d'un blaireau en train d'agrandir son terrier à trente pas d'ici, le minuscule crissement des dents d'un loir adulte entamant la base du champignon sur lequel évoluait la limace. (Folco, Un loup est un loup, Points Seuil : 327s)

Les deux adverbes modalisant la perception auditive indiquent la richesse cognitive de la perception: non seulement parce que chaque bruit est reconnu, rapporté à sa source, y compris en dépit de ses caractéristiques « minuscules » mais, surtout, parce que ces reconnaissances sont quasi simultanées (et, peut-on penser, quasi immédiates aussi), comme les compléments circonstanciels initiaux le donnent à entendre. Autrement dit, « entendre simultanément», associé à l'incrémentation de "presque », campe d'emblée un sujet percevant actif, une perception physiologique et épistémique.

C'est aussi le cas dans la proposition en italique, en (23), qui accompagne la perception en la motivant, en sorte que jeter un coup d'œil, à observer la suite, est un euphémisme et que l'activité perceptive du personnage est très attentive...

(23) Il était près de minuit lorsque Bernard, guidé par le désir de jeter un coup d'oeil à la demeure de son rival, arriva au bas de la rue Norvins. L'endroit était désert. Les gardes mobiles qui stationnaient tout à l'heure sur le trottoir de l'avenue Junot, derrière Le Moulin de la Galette, étaient partis dès après la réunion. Un vent d'automne, humide et quinteux, soulevait des feuilles mortes sur la chaussée. Au carrefour, Bernard hésita une seconde, le temps d'apprécier le ridicule de cette expédition si parfaitement inoffensive. Comme il s'engageait dans la rue Girardon, il vit à l'autre bout surgir une silhouette d'homme qui disparut presque aussitôt par l'escalier descendant à la place Constantin-Pecqueur. De taille moyenne, les épaules larges, la démarche souple, l'inconnu, ainsi vu de dos et à distance, semblait être assez jeune. (Aymé, Travelingue, Folio : 234)

Parfois, le VP est associé à une kyrielle de compléments. Dans l'exemple suivant, les verbes «regarda » sont tous deux associés aux pronoms personnels COD la, le, en (24). Ce qui est très significatif, d'un point de vue sémantique et textuel, avec les informations fournies dans des circonstancielles, des relatives et des complétives, ce sont les qualifications du regard, moins que la cible, en sorte que le sens du VP porte moins sur sa dimension active et intentionnelle que sur une mise en spectacle du regard amoureux, de ses impatiences - tant du point de vue des sujets percevants amoureux que de celui du narrateur - : double PDV que l'on retrouve fréquemment avec le narrateur hugolien (RABATEL, 2008 : 504-505).

(24) Evariste la regarda avec cet air sombre qui mieux que tous les sourires exprime l'amour. Elle le regarda avec une moue un peu moqueuse qui retroussait ses yeux noirs, et cette expression lui venait de ce qu'elle se savait aimée et qu'elle n'était pas fâchée de l'être et de ce que cette figure-là irrite un amoureux, l'excite à se plaindre; l'induit à se déclarer s'il ne l'a pas encore fait, ce qui était le cas d'Evariste. (France, Les Dieux ont soif, Folio [1912] 1989 : 59-60) 
Le verbe «regarda» correspond à la sous-classe <examen visuel actif> (Regarder 1: "Léa regarde le paysage »); mais il est difficile de ne pas faire le rapprochement avec Regarder 9 ( «Léa a regardé dédaigneusement »), qui renvoie à une activité active sans lien avec la perception visuelle, selon GREZKA (2009 : 194-195). Mais à cela près que les jugements sont associés à l'examen visuel, du point de vue des sujets percevants et aussi du point de vue du narrateur, en sorte que d'emblée la mise en discours de regarder pèse sur la signification comme sur la classification des classes-objets, car il apparait que les compléments circonstanciels jouent un rôle décisif dans la catégorisation, davantage que les compléments essentiels. Autrement dit, il faudrait considérer que regarder fait ici l'ellipse d'une comparative avec un deuxième verbe regarder : «Il la regarda comme regardent ceux qui ont cet air sombre qui mieux que tous les sourires exprime l'amour $\gg . .$.

Dans d'autres cas, inutile de donner des compléments, c'est le tiroir verbal qui fait sens, comme en (25), notamment lorsque l'imparfait donne consistance à une vision itérative qui augmente la force épistémique de la perception - phénomène que j'ai analysé dans RABATEL (2008 : 305-321), aussi ne développé-je pas :

(25) Avant que le jour fût levé, Grange entendait sous ses fenêtres le pétillement d'un grand feu de fagots qu'Olivon allumait chaque matin sous sa lessiveuse pour faire fondre la neige (Gracq, Un balcon en forêt, apud MAËSO, 2014 : 143)

De même avec des modalisations du VP, en (26) :

(26) Vers le midi, il s'endormit couché à plat ventre dans un creux du sable, la tête contre le bras, sa loupe tombée de sa main reposant sous lui sur une touffe sèche. Au réveil, il crut apercevoir contre son visage une bête extraordinairement mobile, insecte ou mollusque qui bougeait dans l'ombre. Sa forme était sphérique ; sa partie centrale, d'un noir brillant et humide, s'entourait d'une zone d'un blanc rosâtre ou terne ; des poils frangés croissaient sur la périphérie, issus d'une sorte de molle carapace brune striée de crevasses et bossuée de boursouflures. Une vie presque effrayante habitait cette chose fragile. En moins d'un instant, avant même que sa vision pût se formuler en pensée, il reconnut que ce qu'il voyait n'était autre que son œil reflété et grossi par la loupe, derrière laquelle l'herbe et le sable formaient un tain comme celui d'un miroir. Il se redressa tout songeur. Il s'était vu voyant ; échappant aux routines des perspectives habituelles, il avait regardé de tout près l'organe petit et énorme, proche et pourtant étranger, vif mais vulnérable, doué d'imparfaite et pourtant prodigieuse puissance, dont il dépendait pour voir l'univers. (M. Yourcenar, L'œuvre au Noir, Folio : 242)

Evidemment, ce ne sont pas toutes les modalisations qui intensifient la dimension cognitive. Quoi qu'il en soit, les exemples précédents permettent de dégager l'aspect inhérent lié à la valeur sémantique des prédicats verbaux et à la morphologie verbale ${ }^{18}(25)$ des valeurs aspectuelles contextuelles (Aktionsart) marquées par le lexique, en l'occurrence la modalisation (26) les groupes prépositionnels (23) ou les propositions diverses (24) qui jouent un rôle de prédicat de second ordre, i.e. de prédications secondes (GREZKA, 2009 : 34).

Tout ce qui précède, sans rejeter la nécessité de modéliser, souligne la difficulté de le faire, si on pense dans un cadre textuel. Car pour mener à bien la modélisation,

18 Aspects intemporel, duratif (borné, non borné), inchoatif, progressif, itératif, conclusif, ponctuel/global, sécant... 
on reste dans le cadre de la phrase, alors que le sens déborde la syntaxe phrastique. A. GREZKA reconnaît que les modélisations du sens, notamment celles qui visent le traitement automatique, ne prennent pas en compte la situation, l'identification loc/alloc, la connaissance du monde, les inférences, les intentions, tous ces paramètres importants excédant les capacités des logiciels actuels (GREZKA, 2009 : 20). Si on va en ce sens, alors les barrières deviennent poreuses, ou, dit en d'autres termes, la zone de recouvrement entre VP passifs et VP actifs tend à s'élargir. C'est d'ailleurs ce que GREZKA reconnait dans la fin de son ouvrage, lorsqu'elle note que la prise en compte des éléments contextuels ( $\mathrm{du}$ « co-texte large, assimilateur ou dissimilateur $»^{19}$ ), au-delà de la structure argumentale du verbe (GREZKA, 2009 : 254-255), entraine une zone de recouvrement sémantique entre $\mathrm{V}$ de perception passive et $\mathrm{V}$ de perception active (GREZKA, 2009 : 257). Mais GREZKA limite me semble-t-il ses remarques au cadre de la phrase contenant le VP. Il faudrait pouvoir aller au-delà, parce que le moment perceptif, tant au plan anthropologique qu'au plan discursif, ne porte pas toujours, tant s'en faut, au seul verbe de perception et à ses compléments dans le cadre de la phrase.

La conclusion que l'on peut tirer de ce qui précède, au plan énonciatif et dialogique/polyphonique, au-delà des analyses sémantiques, macro-syntaxiques ou lexicales précédentes, c'est que le sujet du VP dans le cadre des CRP est dans la plupart des cas une sorte de sujet modal, dans la mesure où la description de l'objet perçu correspond à la façon de voir du sujet, ce qui pose la question de la prise en charge des processus perceptifs et des autres procès cognitifs ainsi que des valeurs axiologiques, intersubjectives qui leur sont associées (PEC).

\section{Quelques aperçus de la complexité de la prise en charge dans les hétéro- perceptions}

L'idée générale que je voudrais défendre ici est que la prise en charge n'est pas réservée au locuteur/énonciateur premier, que l'énonciateur second peut aussi le faire, comme l'ont laissé entrevoir certaines analyses antérieures. Ce qui présuppose un tout autre cadre conceptuel que celui qui est en principe proposé pour rendre compte et de la notion de prise en charge et des instances susceptibles d'en être à la source.

La PEC correspond au fait, pour l'énonciateur, d'assumer comme vraie(s) une (ou plusieurs) proposition(s) prédiquée(s), à la condition de ne pas réduire le vrai à la vériconditionnalité (ou à la conformité entre le contenu de la proposition et la réalité extralinguistique) : est vrai aussi ce qui est vrai pour le sujet modal en discours (je-vrai, tu-vrai, on-vrai, « fantôme de la vérité » (BERRENDONNER, 1981), qui correspond à l'effacement énonciatif) - même si par ailleurs ce que l'énonciateur croit vrai peut être faux; est vrai enfin tout ce qui correspond aux valeurs modales (en appui sur toutes les modalités, pas seulement sur les modalités aléthiques, donc y compris les modalités intersubjectives), qui correspondent à des façons de voir du locuteur/énonciateur primaires (L1/E1) ou des sujets seconds

\footnotetext{
${ }^{19}$ Notions empruntées à FUCHS $(1994,1996)$.
} 
représentés par L1/E1, 12/e2 ou e2 (RABATEL, 2009 ; 2012a), valeurs modales dont on a vu l'importance, dans les exemples (12), (14), (17), (18) notamment.

Contrairement aux approches traditionnelles de la PEC (voir RABATEL, 2012a), je n'oppose pas la PEC à la non PEC, mais la PEC directe de L1/E1 à la (quasi) PEC indirecte par une autre instance de validation que L1/E1 (dans le hic et nunc de l'énonciation), soit L1'/E1', ou $12 / \mathrm{e} 2$ ou e2, et c'est ensuite dans une deuxième étape que j'analyse si L1/E1 marque son accord, son désaccord avec le PDV d'un autre, ou se contente de le prendre en compte. Les positionnements d'accord, de désaccord ou de neutralité concernent en effet autant l'autre que soi que l'autre de soi $^{20}$ :

\begin{tabular}{|c|c|c|c|}
\hline Prise en charge & \multicolumn{3}{|c|}{$\begin{array}{l}\text { Quasi-prise en charge imputée par L1/E1 à 12/e2 ou à e2 ou } \\
\text { à }(L 1 ') / E 1 \text { ' dans un autre univers... }\end{array}$} \\
\hline$\frac{\text { Instance de prise en }}{\text { charge }(L 1) / E 1}$ & \multicolumn{3}{|c|}{ Instance de validation $12 / e 2$, e2 ou E1' } \\
\hline \multirow[t]{2}{*}{$\begin{array}{c}\text { Syncrétisme des } \\
\text { instances de PEC et } \\
\text { de validation } \\
\text { [Auto-PDV (PDV de } \\
\text { L1/E1)] }\end{array}$} & \multicolumn{3}{|c|}{$\begin{array}{l}\text { Disjonction présupposée des instances de PEC et de validation } \\
\text { [hétéro-PDV (d'un interlocuteur, d'un tiers, de la doxa, voire de } \\
\text { (L1')/E1' dans un autre univers...)] } \\
{\left[\text { autres que soi... et... autres de } \mathrm{soi}^{21}\right]}\end{array}$} \\
\hline & $\begin{array}{c}\text { Accord } \\
=>\text { Fusion } \\
\text { hétérodialogique des } \\
\text { instances de validation } \\
\text { de E1 et e2 ou E1' }\end{array}$ & $\rightarrow$ Prise en compte 4 & $\begin{array}{l}\longrightarrow \text { Désaccord } \\
\Rightarrow>\text { Refus de la } \\
\text { (con)fusion des } \\
\text { instances de } \\
\text { validation de E1 et e2 } \\
\text { ou E1, }\end{array}$ \\
\hline
\end{tabular}

Dès lors, la question qui se pose est de savoir à qui référer telle dimension cognitive, telle valeur modale, telle émotion, telle disposition à agir, telle ou telle inférence liée à une perception sensorielle donnée, autrement dit à l'énonciateur primaire, ou à des énonciateurs seconds, voire aux deux (dans des proportions à déterminer). Ainsi, dans les exemples (12) à (26), c'est le narrateur, L1/E1 qui impute une quasi-prise en charge de la perception aux différents énonciateurs seconds, même si, par endroits ((24), (26)), la présence du narrateur est si sensible qu'on ne peut écarter l'hypothèse d'une double PEC, E1 marquant son accord avec le PDV de e2.

On peut illustrer rapidement le problème à partir d'un énoncé tel que

(27) Pierre ne vit pas Paul arriver,

\footnotetext{
${ }^{20} \mathrm{Je}$ ne traite pas ici des relations de second niveau, accord, désaccord, prise en compte : voir RABATEL (2012b).

${ }^{21}$ Les « autres de soi » correspondent aux diverses positions énonciatives que l'énonciateur premier adopte lorsqu'il envisage les choses de tel ou tel point de vue, dans tel ou tel cadre spatio-temporel, tel ou tel cadre de référence: ainsi, l'énonciateur dialogue avec des «autres » de lui-même, codés (L1'/)E1'. Cet autodialogisme gagne à être distingué de l'hétérodialogisme, par lequel l'énonciateur premier dialogue avec des interlocuteurs ou des tiers (des « autres que soi », codés 12/e2).
} 
dans lequel, bien que Pierre soit le sujet du verbe de perception, il ne peut pas être la source énonciative de la perception, ni la prendre en charge, compte tenu du fait que le procès de perception est nié. La source s'avère donc être, en l'occurrence, le locuteur/énonciateur premier. En revanche, si la négation était à droite du verbe, comme en (28),

(28) Pierre vit que Paul n'arrivait pas,

la perception serait bien celle de Pierre, car ce qui est nié n'est pas l'acte de perception lui-même, mais renvoie à une expérienciation négative effectuée par Pierre, et dont le lecteur est informé à travers lui ${ }^{22}$.

Les modalités extra-prédicatives (GUIMIER, 1989) (extrinsèques), dans le modus, sont plutôt speaker-oriented (BYBEE et al., 1994: 176ss), tandis que les modalités intra-prédicatives (intrinsèques), dans le dictum, sont de type agentoriented (BYBEE et al., 1994 : 176ss) : les premières sont donc prises en charge par L1/E1, tandis que les secondes peuvent l'être par un agent interne à l'énoncé (en l'occurrence, 12/e2). C'est du moins la conclusion de GoSSELIN (2015), même s'il ne la formule pas en ces termes. Cependant, tous les énoncés, comme on peut le voir dans les exemples antérieurs, sont loin de comporter un modus extra-prédicatif plus un dictum comme dans « il est possible que Pierre ait vu/ait cru voir Paul qui courir » ou «Peut-être que Pierre a vu Paul courir», dans lesquels la prise en charge de la modalité renvoie à L1/E1. En l'absence de modus extra-prédicatif, la modalité intraprédicative, qui comprend un modus interne, peut s'accompagner d'une interprétation en appui sur la lecture de re, mais elle peut aussi, suivant les cas, intégrer une lecture speaker-oriented équivalente à une modalité extra-prédicative, de dicto. C'est le cas des énoncés ci-dessous.

$\mathrm{Si}$, en (29) et (30), il n'y a pas de difficulté à poser que «Je» ou «Pierre » prennent en charge le COD, il en va différemment si on insère un verbe modal comme en (29a) et (30a) :

(29) Je vis Paul qui courait

(29a) J'ai cru voir Paul qui courait/courir ${ }^{23}$

\footnotetext{
22 GREZKA propose une analyse similaire à propos de «Léa ne voit pas l'arbre » : ce qui est nié est « voir l'arbre » et non pas l'activité de voir tout court (2009:99).

${ }^{23}$ Le procès n'a pas la même valeur avec l'infinitif ou dans la relative avec verbe conjugué, qui donne une dimension factuellement plus consistante, plus objective à la perception, en focalisant d'abord sur l'objet perçu puis, dans une prédication seconde, sur l'événement ou l'action (LABELLE, 1996 ; Muller, 1995). Voir aussi supra, note 15, l'analyse de Marsac. Un des relecteurs objecte que l'exemple (29a), et avec lui tous ceux qui comprennent une relative de perception avec un verbe modal sont impossibles en français, et devraient donc être précédés d'un astérisque. Selon KLEIBER (1988 : 90-93), la proposition infinitive avec compte rendu de perception (ICP) peut décrire un événement prenant place dans un monde possible potentiel ou contrefactuel, alors que la perception et l'événement perçu dénoté par la relative avec compte rendu de perception (RCP) ne peuvent prendre place que dans le monde réel, factuel, contingent. Cette contrainte par les mondes possibles s'explique d'abord par le fait que l'infinitif, en tant que forme verbale non tensée, exprime "un événement détaché de ses coordonnées temporelles, donc déplaçable dans un monde possible », à la différence de la RCP, « qui, par son verbe conjugué, ancre nécessairement l'événement dans la temporalité » (MARSAC, 2006 :
} 
(30) Pierre vit Paul qui courait/courir

(30a) Pierre crut/a cru voir Paul qui courait/courir

En effet, en (29a) la source modale est L1/E1 ; mais il est plus difficile de dire, hors contexte, si, en (30a), l'instance de prise en charge est e2, ce qu'explicite la reformulation de (30a) en (30a'),

(30a') Pierre crut voir Paul qui courait; en fait, il s'aperçut très vite qu'il ne s'était pas trompé.

ou si, au contraire, il s'agit de L1/E1, comme en (30a') :

(30a') Pierre crut voir Paul qui courait, jamais il n'eut conscience que c'était impossible, Paul étant à l'étranger à cette époque.

La différence tient à des données épistémiques, en principe fournies par le co(n)texte, qui, en ce cas, désambiguïse l'énoncé et la prise en charge ${ }^{24}: c^{\prime}$ est le cas en (26), où il ne fait aucun doute que celui qui croit voir est bien le personnage, et non le narrateur. D'où, par voie de conséquence, les limites d'une réflexion qui ne porte que sur des énoncés isolés (attestés ou non, c'est encore un autre problème), et le caractère parfois illusoire d'une restriction à un énoncé pour isoler les paramètres qui viendraient perturber le système, comme si le sens pouvait ignorer l'importance du global, dans la désambiguïsation de données fortement polysémiques en langue.

On observe des hésitations analogues, avec d'autres verbes modaux. Ainsi, dans

166-167), laquelle est simultanée au temps de la principale (KLEIBER, 1988: 91). La deuxième explication est que " étant intrinsèquement limitée à décrire un objet de perception au moment où il est perçu, la RCP ne peut pas permettre le report de la perception de cet objet à un monde possible », ce qui explique l'échec des auxiliaires modaux, outre le futur, la négation, l'interrogation ou la tournure hypothétique : d'où * «Paul doit/peut/veut/croit le voir qui pleure », alors que l'emploi des modaux est possible avec l'ICP : «Paul doit/peut/veut/croit le voir pleurer» (ibid. : 92). J'avoue comprendre le raisonnement, mais butte sur le fait que ces énoncés dits impossibles ne me semblent pas l'être, et à d'autres lecteurs non plus. Je pense que la contrainte qu'évoque Kleiber est juste, pour des énoncés qui ne concernent qu'un seul énonciateur. Mais je m'intéresse aux énoncés polyphoniques (ou dialogiques) avec deux énonciateurs. Un autre argument, plus substantiel, repose sur le fait que, dans les exemples de KLEIBER, voir a le sens d'une saisie perceptuelle directe, hic et nunc (KLEIBER 1988 : 92). Or, le verbe voir, dans les exemples (29a), (30a), (30a'), (30a'), (31), exprime une saisie perceptuelle imaginative, déconnectée du hic et nunc, qui correspond plutôt à se représenter, s'imaginer. Il resterait à préciser la part des auxiliaires modaux dans cette saisie imaginative, mais cela excède le cadre de ce travail. Quoi qu'il en soit, c'est la raison fondamentale pour laquelle je ne fais pas précéder l'exemple (29a) et ses semblables d'un astérisque. D'ailleurs, même si (29a) est plus acceptable avec l'infinitif (comme en témoigne le contrôle par le pronom : «je l'ai cru voir courir » et «j'ai cru le voir courir»/ «je l'ai cru voir qui courait» et «j'ai cru le voir qui courait»), cette différence ne permet pas d'éliminer «J'ai cru voir Paul qui courait» (KLEIBER, correspondance privée). Quant à la dimension dialogique, elle intervient en un second temps, et pèse sur la PEC de ladite perception modalisée.

${ }^{24}$ GosSELIN $(2015: 11)$ rappelle qu' « un même marqueur peut parfois donner lieu, selon les contextes, à des interprétations qui diffèrent en ce qu'elles impliquent un fonctionnement de re ou de dicto ». Les modalités de re sont intra-prédicatives, de dicto, extra-prédicatives. C'est le cas de (31) notamment. 
(31) Pierre dut voir Paul courir/qui courait,

il est possible de considérer que, si «dut» est un déontique, la source est L1/E1 (31a) ou 12/e2 (31b) :

(31a) Pierre dut (il avait l'obligation de/il était dans l'obligation de) voir Paul courir

(31b) Pierre dut (=Pierre dit qu'il a ressenti le devoir/qu'il s'est fait une obligation de) voir Paul courir ».

En (31b), il semble que l'obligation soit comme intériorisée, voire émane du sujet plutôt que d'une instance supérieure ou d'une obligation extérieure. Notre hypothèse est donc différente de celle de GoSSELIN, car, pour lui, pour que L1/E1 prenne en charge l'obligation déontique, il faudrait que ce soit avec une modalité de dicto, comme en (31c) :

(31c) Il était obligatoire que Pierre voie Paul courir.

En revanche, si dut est un épistémique, comme en (31d), le choix est plus restreint : il est possible que la modalisation épistémique concerne le jugement de L1/E1 à propos de la perception de Pierre, et en ce cas le peut-être est pris en charge par L1/E1, comme l'auxiliaire modal :

(31d) Pierre dut/a dû voir Paul courir = il a pu (peut-être) voir Paul courir.

En ce cas, la modalité intra-prédicative de (31d), de re, est prise en charge par L1/E1 au même titre que si on avait une modalité de dicto, extra-prédicative (31e) :

(31e) Peut-être que Pierre a dû voir Paul courir

De telles questions, portant sur la prise en charge, rejaillissent sur la dimension active/intentionnelle des verbes de perception. Le repérage de la source de PEC dépend ici fortement du sémantisme du verbe modal, et n'admet pas forcément les mêmes paraphrases : ainsi, (32) peut être paraphrasé en (32a) plutôt par " s'il en a l'autorisation », voire «puisque je lui ai donné l'autorisation », si l'instance de prise en charge est plutôt L1/E1 ; en revanche, si l'instance de PEC est Pierre luimême (32b), on (ou je ?) est plutôt enclin à penser, hors contexte, que Pierre à la possibilité de voir le président indépendamment du fait de subordonner cette possibilité à une autre instance extérieure (et supérieure) à Pierre. De même en (33) : si c'est Pierre qui sait comment voir le président, cela découle d'un savoir qui lui est propre, et c'est lui qui en (33a) prend en charge le VP, tandis que si « sait comment voir » est pris en charge par L1/E1, alors il s'agit d'un commentaire de L1/E1 sur Pierre, qui peut être glosé par exemple par (33b) :

(32) Pierre peut voir le président = l'avis peut être pris en charge par L1/E1 et par e2 
(32a) Pierre peut voir le président $=$ Pierre peut le voir s'il a l'autorisation : l'avis est pris en charge par L1/E1 plutôt que par e2 = (Je suis) D'accord pour que Pierre voie le président

(32b) Pierre peut voir le président (s'il le veut) = le jugement est pris en charge par e2, mais n'exclut pas formellement L1/E1 = s'il est d'accord, moi aussi. Autrement dit, la modalité de re est compatible avec une prise en charge de L1/E1, comme si l'on avait une modalité de dicto du type : D'accord, Pierre peut voir le président.

(33) Pierre sait comment voir le président rapidement $=$ PEC de L1/E1 ou de e2

(33a) Pierre sait comment voir le président rapidement $=$ PEC de e $2=$ Pierre n'a besoin de personne pour qu'on l'aide à voir le président. Mais l'hypothèse n'exclut pas formellement une prise en charge de $\mathrm{L} 1 / \mathrm{E} 1$ = Pierre nous a démontré $\mathrm{X}$ fois qu'il savait comment... Autrement dit, là encore, la modalité de re de (33a) est compatible avec une prise en charge de L1/E1, comme s'il y avait une modalité de dicto du type : Assurément, indubitablement, Pierre sait comment voir le président rapidement.

(33b) Ce gars-là est efficace, il sait comment voir le président rapidement $=(\mathrm{PEC}$ de e2 $)+$ jugement de L1/E1 pris en charge par L1/E1

Bref, lorsque les énoncés ne comprennent pas de modus extra-prédicatif, il n'est pas forcément besoin que la modalité soit de dicto, extra-prédicative pour qu'une lecture speaker-oriented se cumule avec une modalité agent-oriented ${ }^{25}$. Quel que soit leur caractère conjectural, ces paraphrases différentes montrent comment le sens (et ses enjeux) sont différents selon que la source de la PEC est l'instance externe ou une instance seconde représentée par L1/E1. Compte tenu de ce qui précède, les informations contextuelles relatives à la détermination de l'instance de PEC se trouvent et dans la proposition contenant le VP et dans l'ensemble des propositions qui relèvent du CRP, quel que soit leur mode de rattachement syntaxique-textuel à la proposition contenant le VP. C'est ce que montrent les propositions « En moins d'un instant, avant même que sa vision pût se formuler en pensée, il reconnut que ce qu'il voyait n'était autre que son œil reflété et grossi par la loupe, derrière laquelle l'herbe et le sable formaient un tain comme celui d'un miroir. Il se redressa tout songeur », en (26), qui confirment bien la quasi-PEC de la perception au compte de l'instance seconde.

Ces questions sont particulièrement importantes pour l'interprétation des VP dans le cadre des CRP. Mais il serait illusoire de croire que la question interprétative est exogène aux problématiques linguistiques «pures et dures ». C'est donc la description, l'analyse linguistique des formes qui est d'emblée confrontée (ou devrait se confronter d'emblée) à la saisie des paramètres pragma-énonciatifs de la perception (entre autres), dans les textes, en situation - d'où l'intérêt stratégique des questions de prise en charge des valeurs modales et du jeu des paraphrases - si l'on veut bien considérer la perception comme un processus complexe qui fait sens dans la dynamique discursive, qui doit être articulé avec les pensées/paroles et actions (ce que je propose dans RABATEL (2008: 440-446, 464-466), à partir de la

${ }^{25}$ L'analyse pourrait être complétée avec un autre critère, celui des énoncés montrés véridicibles $v s$ non véridicibles : voir KRONNING (2013) et Gosselin (2015: 12). Cette question fera l'objet d'un examen ultérieur. 
problématique de la pré-réflexivité à la réflexivité) au plan ontologique/phénoménologique d'une part, et textuel d'autre part.

\section{BIBLIOGRAPHIE}

BENZAKOUR Fouzia (1990), Les compléments de comptes rendus de perception : quelques cas en français, Thèse de doctorat, nouveau régime, Université de Strasbourg.

BANFIELD Ann (1982,1995), Phrases sans parole. Théorie du récit et style indirect libre, Paris, Le Seuil.

BERRENDONNER Alain (1981), Eléments de pragmatique linguistique, Paris, Éditions de Minuit.

BERTHOZ Alain (2004), Physiologie du changement de point de vue, in : BERTHOZ Alain et JORLAND Gérard (dir), L'empathie, Paris, Odile Jacob, p. 253-275.

BRUNEL Marie-Lise ; CosNier Jacques (2012), L'empathie. Un sixième sens, Lyon, Presses universitaires de Lyon.

BybeE Joan; PERKIns Revere; PAGLIUCA William (1994), The Evolution of Grammar, Chicago, C.U.P.

Conche Marcel (2016), Penser encore. Sur Spinoza et autres sujets, Paris, Les Belles Lettres.

DendALE Patrick ; TASMOVSKi Liliane (1994), L'évidentialité ou le marquage des sources du savoir, Langue française 102, p. 3-7.

Dubois Danièle (dir) (2009), Le sentir et le dire. Concepts et méthodes en psychologie et en linguistique cognitive, Paris, L'Harmattan.

DUCROT Oswald (1984), Le dire et le dit, Paris, Éditions de Minuit.

GoSSELIN Laurent (2010), Les modalités en français. Amsterdam, New York, Rodopi.

GosSELIN Laurent (2015), De l'opposition modus / dictum à la distinction entre modalités extrinsèques et intrinsèques, Bulletin de la société de linguistique de Paris, T. CX, fasc. 1, p. 1-50.

GreZKA Aude (2009), La polysémie des verbes de perception visuelle, Paris, L'Harmattan.

Gross Gaston (2015), Statut de la sémantique, in : RABATEL Alain, FerRARALETURGIE Alice; LETURGIE Arnaud (éds), La sémantique et ses interfaces, Éditions Lambert-Lucas, p. 85-110.

GuentCheVA Zlatka (1996), Énonciation médiatisée, Paris, Louvain, Peeters.

GUIMIER Claude (1989), Constructions syntaxiques et interprétations de pouvoir, Langue française 84, p. 9-23.

JoUvenT Roland [2009] (2013), Le cerveau magicien. De la réalité au plaisir psychique, Paris, Odile Jacob.

KLEIBER Georges (1988), « Sur les relatives du type Je le vois qui arrive », Travaux de linguistique, 17, p. 89-115.

KLEIBER Georges (2011), Pour une linguistique des odeurs: présentation, Langages 181, p. 3-15.

KRONNING Hans (2013), Monstration, véridiction et polyphonie. Pour une théorie modale de la polyphonie, in: CONSTANTIN DE ChANAY Hugues; COlAS- 
Blaise Marion; Le Guern Odile (éds), Dire/montrer. Au cœur du sens, Chambéry, Éditions de l'Université de Savoie, Collection Langages, p. 93-115.

LABELle Marie (1996), Remarques sur les verbes de perception et la souscatégorisation » Recherches linguistiques de Vincennes, 25, p. 83-106.

Livet Pierre (éd.) (2000), De la perception à l'action. Contenus perceptifs et perception de l'action, Paris, Vrin.

MAËSO Florence (2014), Etude linguistique des comptes rendus de perception dans le contexte des $N$ fenêtre(s) et porte(s) : approche sémantique et pragmatique, Thèse de doctorat, université de Paris 3 .

MARSAC Fabrice (2006), Les constructions infinitives régies par un verbe de perception, Thèse de doctorat, université de Strasbourg.

MARSAC Fabrice (2010), Du statut fonctionnel de l'infinitif régi par un verbe de perception, in: CASTRO Camino Álvarez; BANGO de la CAMPA Flor $\mathrm{M}^{\mathrm{a}}$; DONAIRE Maria Luisa (éds), Liens linguistiques. Etudes sur la combinatoire et la hiérarchie des composants, Berne, Peter Lang, p.165-184.

MULLER Claude (1995), Les relatives de perception: J'entends le garçon qui bégaie qui bégaie, in: BAT-ZEEV-SHYLDKROT Hava; KUPFERMAN Lucien (éds), Tendances récentes en linguistique française et générale, volume dédié à David Gaatone, Amsterdam, Benjamins, p. 310-322.

OLSEN Michel (2002), Le passé simple subjectif, Polyphonie-linguistique et littéraire 4, Université de Roskilde, Danemark, p.101-123.

RABATEL Alain (1997), Une histoire du point de vue, Metz, CELTED/Klincksieck.

RABATEL Alain (1998), La construction textuelle du point de vue, Paris, Lausanne, Delachaux et Niestlé.

RABATEL Alain (2003), Les verbes de perception en contexte d'effacement énonciatif: du point de vue représenté aux discours représentés, Travaux de linguistique 46, p. 49-88.

RABATEL Alain (2008), Homo narrans. Pour une analyse énonciative et interactionnelle du récit. Tome 1. Les points de vue et la logique de la narration. Tome 2. Dialogisme et polyphonie dans le récit, Limoges, Éditions LambertLucas.

RABATEL Alain (2009), Prise en charge et imputation, ou la prise en charge à responsabilité limitée, Langue française 162, p.71-87.

RABATEL Alain (2010), Retour sur les relations entre locuteur et énonciateur. Des voix et des points de vue, in: COlAS-BLAISE Marion; KARA Mohamed; PERRIN Laurent; PETITJEAN André (éds), La question polyphonique ou dialogique dans les sciences du langage, Collection Recherches linguistiques 31, Metz, CELTED, Université de Metz, p. 357-373.

RABATEL Alain (2012a), Sujets modaux, instances de prise en charge et de validation, Le discours et la langue 3-2, p.13-37.

RABATEL Alain (2012b), Positions, positionnements et postures de l'énonciateur, Travaux neuchâtelois de linguistique 56, p. 23-42.

RABATEL Alain (2014), Empathie, points de vue, méta-représentation et dimension cognitive du dialogisme, Études de linguistique appliquée 173, p. 27-45.

RABATEL Alain (2015), D'un mode de signifiance sémantique pathémique-iconique fréquent en poésie à un mode d'énonciation subjectif-empathique, in : 
BedouRET Sandrine; LAPlantine Chloé (éds), Émile Benveniste : vers une poétique générale, Pau, Presses Universitaires de Pau et des Pays de l'Adour, p. 111-137.

RABATEL Alain (2016), Diversité des points de vue et mobilité empathique, in : COlAS-Blaise Marion; Perrin Laurent; TORE Gian Maria (éds), L'énonciation aujourd'hui, un concept-clé des sciences du langage, Limoges, Éditions Lambert-Lucas, p. 137-152.

RoIG Audrey; VAN RAEMDONCK Dan (éds) (2014), Les liaisons de prédications. Langue française 182.

RoIG Audrey; VAn RAEMdonck Dan (2014), Insubordinons-nous! Lecture critique de la subordination, Langue française 182, p. 11-25.

RoIG Audrey; VAN RAEMDONCK Dan (éds) (2015), Les liaisons de prédications : lorsque la marque s'estompe, Langages 200.

RASTIER François (2015), Saussure au futur, Paris, Les Belles Lettres.

SAUSSURE Ferdinand de (2002), Écrits de linguistique générale, Paris, Gallimard.

TiSSERON Serge (2010), L'empathie au cour du jeu social, Paris, Albin Michel.

UTAKER Arild [2013] (2016), Le retour de Saussure, in : RASTIER François (dir), De l'essence double du langage et "le renouveau du saussurisme », Limoges, Éditions Lambert-Lucas, p. 95-106.

VERINE Bertrand (2014), Dire le non visuel, Liège, Presses universitaires de Liège. 